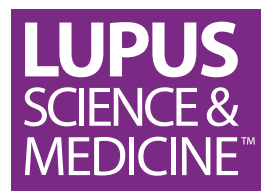

\title{
Disease and economic burden increase with systemic lupus erythematosus severity 1 year before and after diagnosis: a real-world cohort study, United States, 2004-2015
}

\author{
Miao Jiang, ${ }^{1}$ Aimee M Near, ${ }^{2}$ Barnabas Desta,${ }^{1}$ Xia Wang, ${ }^{1}$ Edward R Hammond ${ }^{1}$
}

To cite: Jiang M, Near AM, Desta B, et al. Disease and economic burden increase with systemic lupus erythematosus severity 1 year before and after diagnosis: a real-world cohort study, United States, 2004-2015. Lupus Science \& Medicine 2021;8:e000503. doi:10.1136/ lupus-2021-000503

- Additional supplemental material is published online only. To view, please visit the journal online (http://dx.doi.org/10. 1136/lupus-2021-000503).

Data were presented at the American College of Rheumatology/Association of Rheumatology Professionals (ACR/ARP) 2019 Annual Meeting, Atlanta, GA, 8-13 November 2019.

Received 22 March 2021 Accepted 19 August 2021

Check for updates

(C) Author(s) (or their employer(s)) 2021. Re-use permitted under CC BY-NC. No commercial re-use. See rights and permissions. Published by BMJ.

${ }^{1}$ BioPharmaceuticals Medical, AstraZeneca, Gaithersburg, Maryland, USA

${ }^{2}$ Real-World Evidence, IQVIA, Durham, North Carolina, USA

Correspondence to Barnabas Desta; Barnabas. Desta@astrazeneca.com

\section{ABSTRACT}

Objective To assess the economic burden of patients with SLE by disease severity in the USA 1 year before and after diagnosis.

Methods Patients aged $\geq 18$ years with a first SLE diagnosis (index date) between January 2005 and December 2014 were identified from administrative commercial claims data linked to electronic medical records (EMRs). Disease severity during the year after diagnosis was classified as mild, moderate, or severe using claims-based algorithms and EMR data. Healthcare resource utilisation (HCRU) and all-cause healthcare costs (2017 US\$) were reported for 1 year pre-diagnosis and post-diagnosis. Generalised linear modelling examined all-cause costs over 1 year post-index, adjusting for baseline demographics, clinical characteristics, Charlson Comorbidity Index and 1 year pre-diagnosis costs. Results Among 2227 patients, $26.3 \%$ had mild, $51.0 \%$ moderate and $22.7 \%$ severe SLE. Mean per-patient costs were higher for patients with moderate and severe SLE compared with mild SLE during the year before diagnosis: mild US\$12 373, moderate \$22 559 and severe US\$39261 $(p<0.0001)$; and 1-year post-diagnosis period: mild US\$13 415, moderate US\$29 512 and severe US\$68 $260(p<0.0001)$. Leading mean cost drivers were outpatient visits (US\$13 566) and hospitalisations (US\$10 252). Post-diagnosis inpatient utilisation ( $\geq 1$ stay) was higher for patients with severe $(51.2 \%)$ and moderate (22.4\%) SLE, compared with mild SLE (12.8\%), with longer mean hospital stays: mild 0.47 days, moderate 1.31 days and severe 5.52 days $(p<0.0001)$.

Conclusion HCRU and costs increase with disease severity in the year before and after diagnosis; leading cost drivers post-diagnosis were outpatient visits and hospitalisations. Earlier diagnosis and treatment may improve health outcomes and reduce HCRU and costs.

\section{INTRODUCTION}

SLE is a chronic autoimmune disease associated with significant morbidity and mortality, affecting multiple organ systems. ${ }^{12}$ SLE is associated with high annual costs of care that are greater than for some other chronic

\section{Key messages}

What is already known about this subject?

- SLE is associated with significant healthcare resource utilisation (HCRU) and costs, especially during periods of heightened disease activity.

- Patients who receive earlier diagnoses have lower flare rates, less HCRU and lower costs, compared with those who have later diagnoses.

What does this study add?

- This study used administrative commercial claims data linked to electronic medical records to evaluate the economic burden of US patients with newly diagnosed SLE in the 1-year period before and after diagnosis.

- In the year before diagnosis, unadjusted all-cause healthcare costs were 1.8-fold higher for patients with severe SLE and 3.2-fold higher for patients with moderate SLE than for mild SLE, predominantly owing to outpatient visits and hospitalisations.

- In the year post-diagnosis, healthcare costs were 2.2-fold and 5.1-fold for patients with moderate and severe SLE, respectively, compared with mild SLE. Multiple factors, including the presence of $\geq 2$ Charlson Comorbidity Index comorbidities at baseline, the use of $\geq 3$ medications at baseline and higher healthcare costs during the baseline period, are associated with increased healthcare costs during the year after diagnosis.

How might this impact on clinical practice or future developments?

- These findings highlight the importance of early diagnosis and rapid treatment. Early diagnosis and treatment may improve disease control and health outcomes to reduce the economic burden of SLE.

conditions, such as fibromyalgia and rheumatoid arthritis. ${ }^{3-5}$ In a systematic review of SLE healthcare costs and utilisation, mean annual direct costs per patient ranged $\$ 15$ 171-\$88445 (2016 US\$), with the broad 
range underscoring the effect that disease severity can have on overall healthcare costs. ${ }^{3}$

SLE is characterised by episodes of increased disease activity; flares are separated by periods of remission. ${ }^{2}$ Studies have shown that $65 \%-70 \%$ of patients with SLE may experience at least one flare per year. ${ }^{67}$ SLE flares are associated with increased annual medical costs, which increase with flare severity. ${ }^{8-11}$ As there is currently no curative therapy for SLE, one of the main treatment goals is to prevent flares and disease progression. ${ }^{2}$

Current medications approved by the US Food and Drug Administration to treat SLE include corticosteroids, antimalarials such as hydroxychloroquine, and belimumab, a biologic. ${ }^{12-17}$ Other therapies for SLE management include nonsteroidal anti-inflammatory drugs (NSAIDs), immunosuppressive and/or immunomodulatory agents, and rituximab, a biologic. ${ }^{18}$ Although corticosteroids provide clinical benefits, long-term use has been associated with organ damage and toxicity, along with increased healthcare resource utilisation (HCRU) and costs. ${ }^{16} 19-21$

Previous studies demonstrated that SLE disease severity is associated with substantial HCRU and costs. ${ }^{911} 13-151722$ The time from symptom onset to SLE diagnosis can be long, with one study reporting a mean duration of 21.8 months. ${ }^{23}$ Patients who receive earlier diagnoses have lower flare rates, less HCRU and lower costs, compared with those who have later diagnoses. ${ }^{24}$ Given the complexity of SLE disease progression, few studies have quantified the economic burden along the patient journey from the period leading up to diagnosis through post-diagnosis treatment in the USA. Only one study, in a population-based Canadian cohort, has evaluated the economic burden of SLE pre-diagnosis. This study showed an increase in incremental direct medical costs of SLE over the 5 years before diagnosis; however, the results were not stratified by disease severity. ${ }^{25}$

The objective of this study was to assess the economic burden of SLE and its association with disease severity in the year before and after initial diagnosis. We conducted a retrospective study using administrative commercial claims data linked to electronic medical records (EMRs) among a cohort of US patients with newly diagnosed SLE.

\section{PATIENTS AND METHODS}

\section{Data sources}

This retrospective study leveraged the IBM MarketScan commercial database linked to the General Electric Centricity EMR database (GE EMR) with data from January 2004 to December 2015. The IBM MarketScan commercial database contains fully integrated, longitudinal, de-identified, patient-level healthcare claims data on clinical utilisation, expenditures and enrolment across inpatient, outpatient, prescription drug and carve-out services. The data are from large employers, health plans, and government and public organisations and include private sector health data from approximately 350 payers; historically, $>20$ billion service records have been included.

The GE EMR database includes patient-level information on the following: demographics; lifestyle characteristics; insurance coverage; vital signs; International Classification of Diseases, Ninth Revision (ICD-9) and ICD-10 medical diagnoses; patient complaints; diagnostic and laboratory tests with results; procedures; prescriptions; and information from specialty healthcare providers. Clinical data are captured from $>725$ member institutions and 33000 providers and include $>38$ million patients from 49 US states and the District of Columbia.

The study dataset was constructed by linking patient data from IBM MarketScan and GE EMR using a patented and proprietary encryption algorithm developed by IQVIA. ${ }^{26-28}$ Patient data were de-identified across data suppliers using the encryption algorithm, followed by deterministic matching based on patient-level information. Each patient was then assigned a unique and persistent IQVIA patient ID with linkage across various databases.

The study data consist of fully de-identified datasets, in compliance with the US Health Insurance Portability and Accountability Act; therefore, the study did not require Institutional Review Board approval.

\section{Study design and patient selection}

Patients with SLE from the linked dataset were eligible for inclusion if they had at least one SLE diagnosis (ICD-9-CM: 710.0x, ICD-10-CM: M32.9) in EMR records or claims in any position, either as $\geq 1$ inpatient SLE diagnosis or $\geq 2$ separate outpatient diagnoses (including the index diagnosis) that were $\geq 60$ days apart between 1 January 2005 and 31 December 2014. Two medical claims for outpatient settings were required to limit potential misclassification of SLE cases, which tends to be more likely in outpatient settings than inpatient and emergency department (ED) settings. The date of first observed SLE diagnosis was defined as the index date. To further minimise potential misclassification, and confirm patients with SLE, patients were also required to have used SLE-related medications, identified by national drug codes or healthcare common procedure coding system codes in the pharmacy claim, within 6 months before and after the index date (online supplemental table 1). Patients were $\geq 18$ years of age on the index date, with continuous health plan enrolment for at least 12 months pre-index (baseline period) and 12 months post-index (follow-up period). The continuous enrolment requirement ensured that HCRU and costs were comprehensively captured within the data sources. To ensure newly diagnosed, not prevalent SLE cases, patients were excluded if they had a prior diagnosis of SLE or lupus nephritis during the baseline period. Patients were also excluded if their data were incomplete or had other quality issues, such as missing age or sex. Figure 1 presents details of the inclusion and exclusion criteria with attrition of the study population. 


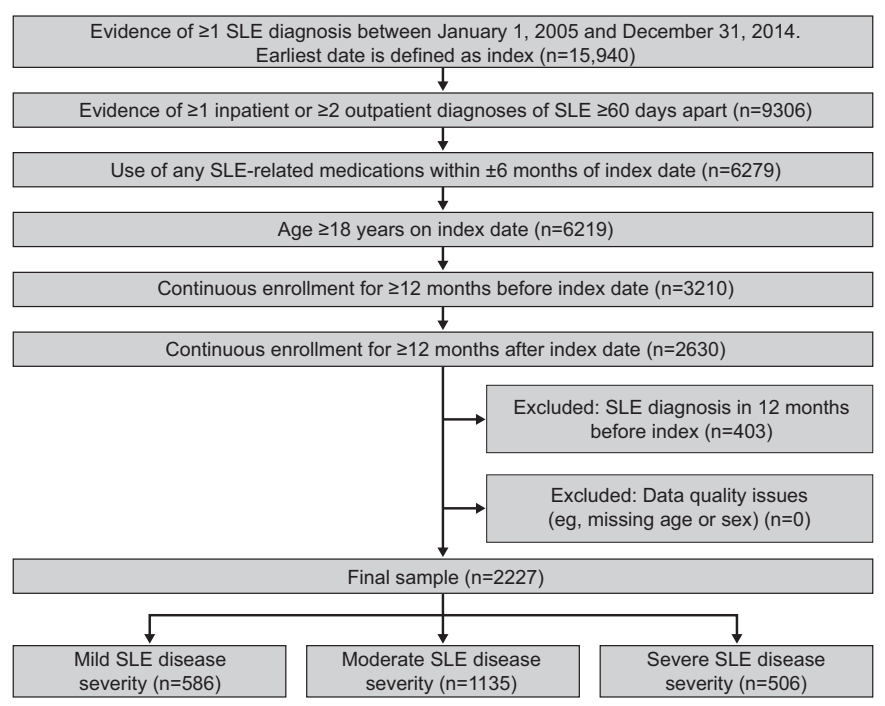

Figure 1 Attrition of the identified study population of US patients with newly diagnosed SLE.

\section{Study measures}

SLE disease severity

Disease severity was classified as mild, moderate or severe based on the highest disease severity experienced over 1 year post-diagnosis using claims-based algorithms, ${ }^{9}$ which combined SLE diagnosis, disease activities and SLErelated conditions, medications and health services use, supplemented with EMR. The algorithms are described in online supplemental table 2 . We chose the 1-year postdiagnosis window because it reflects an accurate and comprehensive view of disease severity, accounting for the variation in the disease process over time while allowing sufficient time for clinical evaluation and diagnosis.

\section{Baseline characteristics}

Baseline demographic characteristics included age, sex, race/ethnicity, geographical region, health plan type and payer type, assessed at the index date. Baseline clinical characteristics included Charlson Comorbidity Index (CCI) score and medication use, assessed over the baseline period. In addition, the proportions of patients with $0,1,2$ and $\geq 3$ CCI comorbidities, individual CCI conditions and SLE-related non-CCI conditions were reported. All-cause healthcare costs as the total payments received by providers, including the amounts paid by payers and patient out-of-pocket cost (eg, copay, co-insurance), converted to 2017 US dollars using the medical component of the Consumer Price Index, were also measured during the baseline period.

\section{Outcome measures}

The study outcomes included all-cause healthcare costs, HCRU and treatment patterns during the 1-year postdiagnosis period, overall and by care setting (inpatient, ED, outpatient, office, laboratory and pharmacy). Healthcare costs were estimated for the 1-year post-diagnosis period; a similar estimate was made for baseline costs. Components of inpatient HCRU assessed included the proportion of patients with $\geq 1$ inpatient hospitalisation, mean number of hospitalisations and mean hospital length of stay. Outpatient, ED, office, laboratory and pharmacy HCRU were assessed as the proportion of patients with $\geq 1$ visit, service or prescription, and the mean number of utilisations for each category. Outpatient services include all nonpharmacy claims not categorised as inpatient, ED, office or laboratory services. Prescribed SLE treatments during the 1-year post-diagnosis period were also assessed. Outcomes were evaluated for all patients and stratified by SLE disease severity.

\section{Statistical analyses}

Baseline patient characteristics and clinical outcomes during the follow-up period were reported as counts or proportions for categorical variables and means and SD for continuous variables. Descriptive comparisons between SLE severity groups were examined with Pearson's $\chi^{2}$ test or F-test for categorical variables and analysis of variance or t-test for continuous variables. A generalised linear model with gamma distribution and log link was fit to evaluate the incremental cost by SLE severity as well as factors associated with total all-cause healthcare cost during the 1-year post-diagnosis period, adjusting for baseline demographic and clinical characteristics, and all-cause healthcare costs during the baseline period. Statistical tests were two-sided with an $\alpha$-level of 0.05 for statistical significance. All analyses were performed with SAS V.9.4 (SAS Institute, Cary, NC, USA).

\section{Patient and public involvement}

Patients and the public were not involved in the research process, research questions, study design, or result dissemination plans.

\section{RESULTS}

\section{Patient demographics and clinical characteristics}

The study population included 2227 patients newly diagnosed with SLE: 586 (26.3\%) with mild SLE, 1135 $(51.0 \%)$ with moderate SLE and $506(22.7 \%)$ with severe SLE. Baseline demographics and clinical characteristics are reported in table 1 . The mean (SD) age of patients was 50.2 (13.0) years, $54.4 \%$ were non-Hispanic white and $90.6 \%$ were female. Overall, $58.5 \%$ of patients were from the South, $18.6 \%$ from the Northeast and $13.0 \%$ from North central US regions. Patients were largely covered by commercial insurance $(87.7 \%)$ and the remaining by employer-provided Medicare supplemental insurance $(12.3 \%)$. Across SLE severity groups, demographics were similar except that patients with severe SLE were more likely to be $>65$ years old, male and covered by Medicare (table 1).

The mean (SD) CCI score at baseline was 1.2 (1.5) for all patients and increased with SLE disease severity: 0.8 (1.1) for mild SLE, 1.1 (1.4) for moderate SLE and 1.8 (1.8) for severe SLE $(p<0.0001)$. The presence of $\geq 1$ CCI comorbidity at baseline was more frequent among patients with severe SLE (73.7\%) and moderate SLE (59.4\%) 
Table 1 Baseline demographics and clinical characteristics ${ }^{\star}$ for patients with newly diagnosed SLE by disease severity

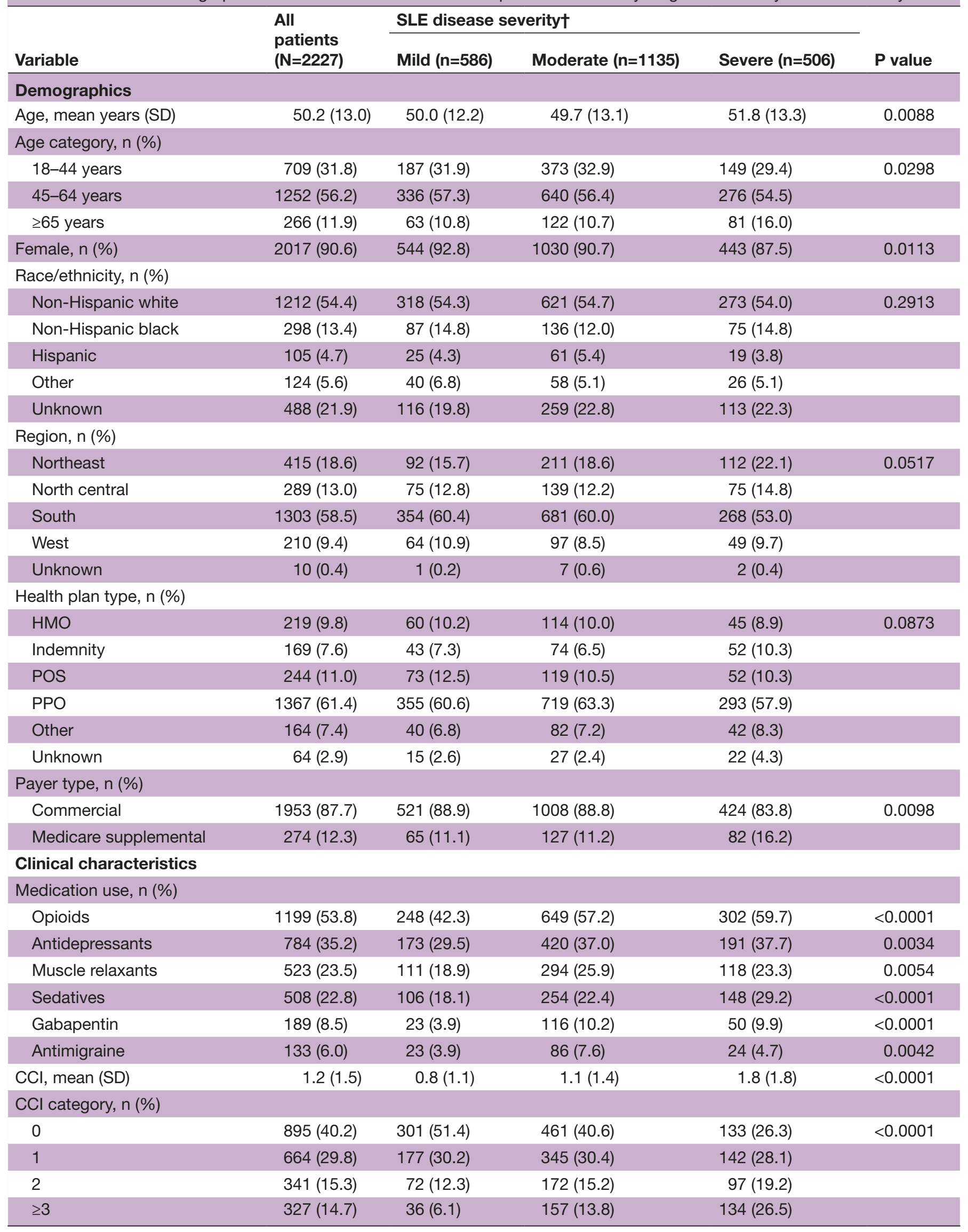


Table 1 Continued

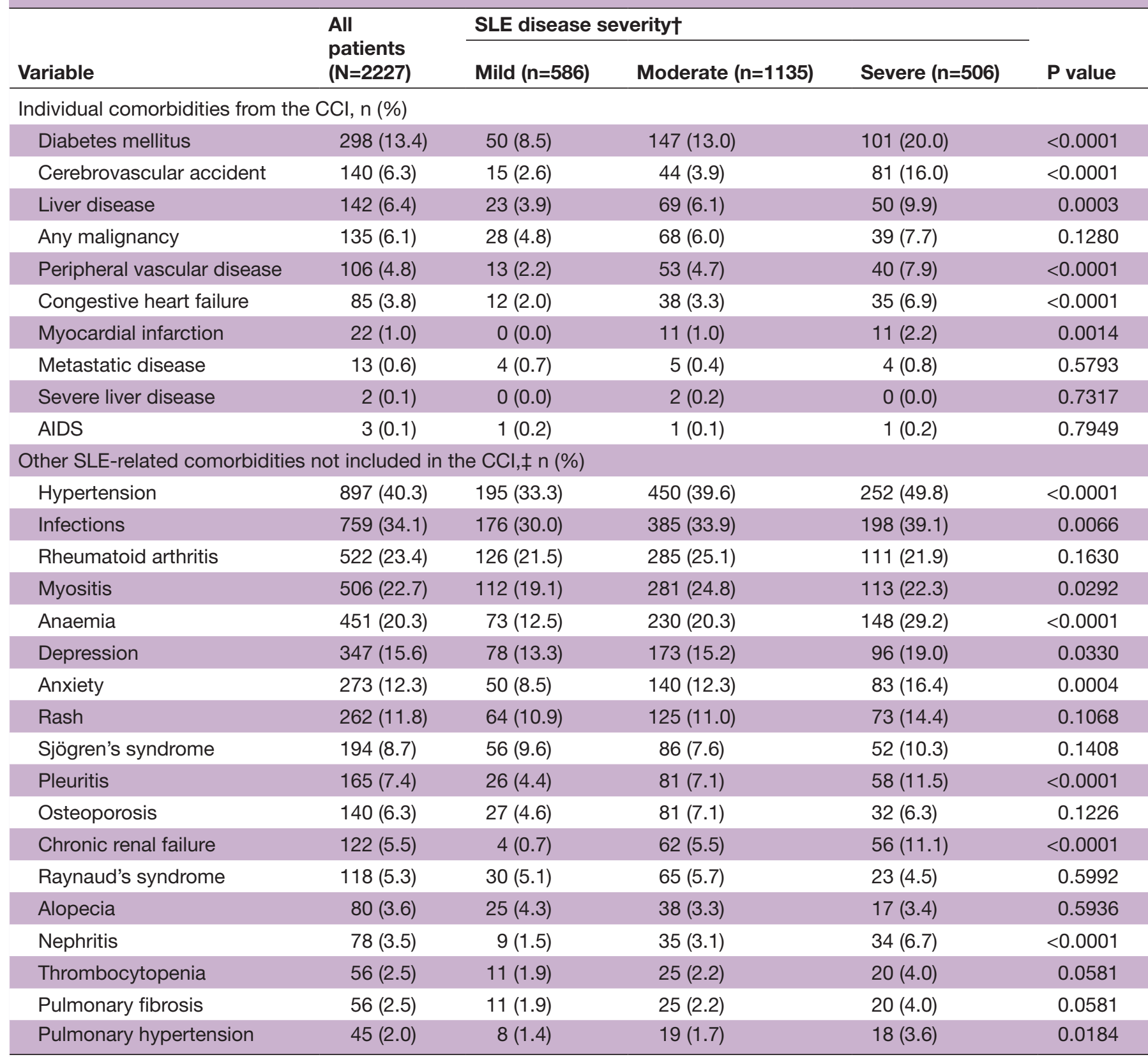

*During the 1-year period before diagnosis.

†Disease severity was assessed during the 1-year period after diagnosis, and patients were classified to the most severe level during that period.

$\ddagger$ SLE-related non-CCI comorbidity reported if $\geq 2 \%$ among all patients.

$\mathrm{CCl}$, Charlson Comorbidity Index; HMO, health maintenance organisation; POS, point of service; PPO, preferred provider organisation.

compared with mild SLE (48.6\%). Patients with severe or moderate SLE had significantly higher frequencies of diabetes mellitus, cerebrovascular accident, liver disease, peripheral vascular disease, congestive heart failure and myocardial infarction, compared with patients with mild SLE (all $\mathrm{p}<0.01$ ). For the top 10 most observed comorbidities not included in the CCI, patients with severe or moderate SLE had significantly higher frequencies of hypertension, infections, myositis, anaemia, depression, anxiety and pleuritis, compared with mild SLE (table 1).
SLE medications prescribed during the 1-year post-diagnosis (follow-up) period

The most commonly prescribed medications during the post-diagnosis period were corticosteroids $(76.1 \%)$, hydroxychloroquine (59.7\%), NSAIDs $(36.7 \%)$ and methotrexate $(14.7 \%$ ) (online supplemental table 3). Biologic drugs, belimumab and rituximab, were prescribed to $1.4 \%$ and $1.3 \%$ of patients, respectively.

Medication use differed with SLE disease severity. Hydroxychloroquine was the most frequently prescribed 
Table 2 Healthcare resource utilisation during the 1-year post-diagnosis (follow-up) period for patients with newly diagnosed SLE by disease severity

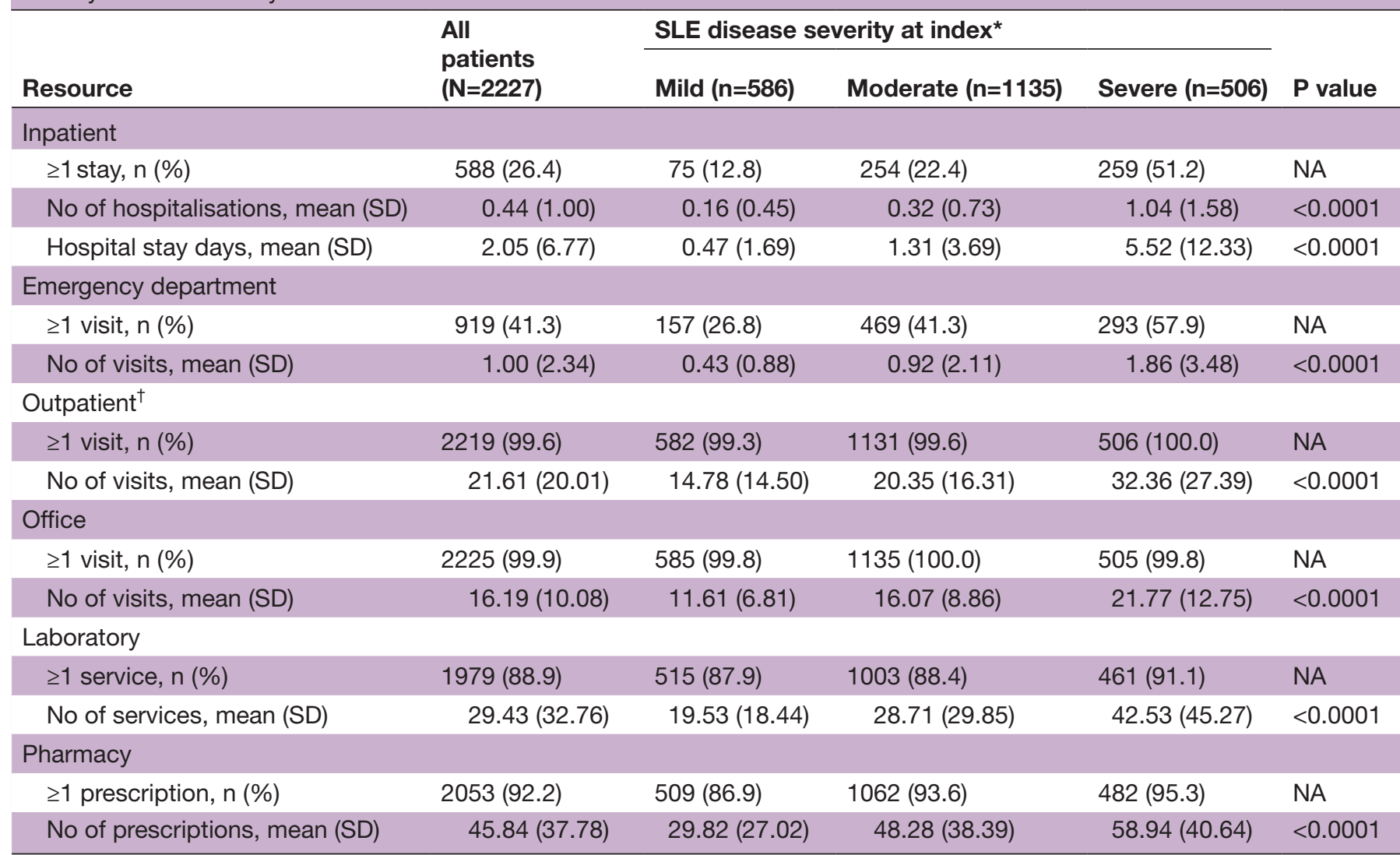

*Disease severity was assessed during the 1-year period after diagnosis, and patients were classified to the most severe level during that period.

†Outpatient services included all nonpharmacy claims not categorised as inpatient, emergency department, office or laboratory services. NA, not assessed.

medication for patients with mild SLE (63.7\%), compared with $61.3 \%$ and $51.6 \%$ for patients with moderate and severe SLE, respectively ( $<<0.0001$ for difference between groups). Corticosteroids were the most frequently prescribed medication for patients with moderate and severe SLE $(87.5 \%$ and $86.2 \%$, respectively), compared with $45.4 \%$ of patients with mild SLE $(\mathrm{p}<0.0001)$. Patients with moderate and severe SLE received more prescriptions for immunosuppressants and biologics compared with patients with mild SLE. Methotrexate, mycophenolate mofetil, azathioprine and cyclophosphamide were prescribed to proportionally more patients with moderate and severe disease compared with mild disease (online supplemental table 3). Prescriptions for belimumab were more frequent among patients with severe $(1.8 \%)$ and moderate SLE (1.9\%) compared with mild SLE $(0.3 \%, \mathrm{p}<0.03)$. A total of $5.7 \%$ of patients with severe SLE received prescriptions for rituximab, compared with no patients with moderate SLE or mild SLE $(p<0.0001)$ (online supplemental table 3).

\section{All-cause HCRU during the 1-year post-diagnosis (follow-up)} period

Overall, $26.4 \%$ of patients with SLE had $\geq 1$ inpatient hospitalisation during the 1-year post-diagnosis period, with a mean (SD) length of stay of 2.05 (6.77) days (table 2). The proportion of patients with $\geq 1$ inpatient hospitalisation increased with disease severity: $12.8 \%$, $22.4 \%$ and $51.2 \%$ for mild, moderate and severe SLE, respectively; as did mean (SD) length of stay, with 0.47 (1.69) days, 1.31 (3.69) days and 5.52 (12.33) days, respectively $(\mathrm{p}<0.0001)$ (table 2$)$. Patients with severe and moderate SLE had a higher mean (SD) number of hospitalisations, with $1.04(1.58)$ visits and $0.32(0.73)$ visits, respectively, compared with 0.16 (0.45) visits for patients with mild SLE $(\mathrm{p}<0.0001)$. Overall, $41.3 \%$ of patients had $\geq 1$ ED visit; $26.8 \%$ of patients with mild, $41.3 \%$ with moderate and $57.9 \%$ with severe SLE had $\geq 1$ ED visit.

Outpatient services ( $\geq 1$ visit) were used by $>99 \%$ of patients, regardless of disease severity. Patients with severe and moderate SLE had a higher mean (SD) number of outpatient visits, 32.36 (27.39) and 20.35 (16.31), respectively, compared with patients with mild SLE, who had 14.78 (14.50) visits $(\mathrm{p}<0.0001)$. Office services were used by $>99 \%$ of patients and laboratory and pharmacy services by $>85 \%$, regardless of disease severity. 

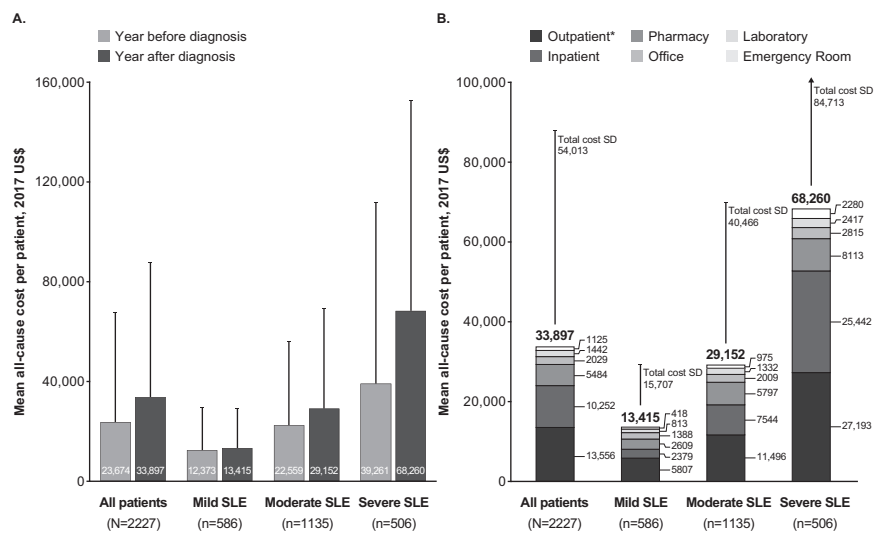

Figure 2 All-cause healthcare costs per patient (A) during the baseline and 1-year post-diagnosis (follow-up) periods for all patients with newly diagnosed SLE and by SLE disease severity and (B) during the 1-year post-diagnosis (follow-up) period for all patients with newly diagnosed SLE, by SLE disease severity and setting. Error bars show SD. A detailed breakdown of costs per care setting is available in online supplementary table 4. *Outpatient services included all nonpharmacy claims not categorised as inpatient, emergency department, office or laboratory services.

\section{All-cause healthcare costs during the 1-year baseline and post-diagnosis (follow-up) periods}

The mean (SD) unadjusted all-cause healthcare costs during the baseline period for patients with newly diagnosed SLE were US\$23674 (US\$44 113). The mean (SD) all-cause costs increased with increasing disease severity: mild SLE US\$12373 (US\$17 171), moderate SLE US\$22559 (US\$33 674) and severe SLE \$39261 (US\$72 768), $\mathrm{p}<0.0001$ (figure 2A). All-cause healthcare costs were 1.8-fold and 3.2-fold higher for patients with moderate and severe SLE, respectively, compared with mild SLE.

The mean (SD) unadjusted all-cause healthcare costs during the 1-year post-diagnosis period were US\$33897 (US\$54 013) for all patients. Healthcare costs increased with increasing SLE severity: US\$13415 (\$15 707) for patients with mild SLE, US\$29152 (US\$40 466) for moderate SLE and US\$68260 (US\$84 712) for severe SLE $(\mathrm{p}<0.0001)$ (figure 2A). This represents a 2.2-fold and 5.1-fold higher healthcare cost for patients with moderate and severe SLE, respectively, compared with mild SLE.

When adjusted for baseline demographics, clinical characteristics and all-cause healthcare costs during the baseline period, increasing SLE disease severity remained associated with increasing healthcare costs (table 3). Moderate and severe SLE was associated with significantly higher total costs, compared with mild SLE (moderate SLE cost ratio (95\% CI): 1.81 (1.65 to 1.98), $\mathrm{p}<0.0001$; severe SLE cost ratio (95\% CI): 4.24 (3.80 to 4.73), $\mathrm{p}<0.0001)$. Other factors associated with higher healthcare costs during the post-diagnosis period include the presence of $\geq 2$ CCI comorbidities at baseline, use of $\geq 3$ medications at baseline and higher healthcare costs during the baseline period (table 3 ).
During the post-diagnosis period, the leading cost driver for all patients was outpatient visits at a mean (SD) cost of US $\$ 13566$ (US $\$ 32747$ ), followed by hospitalisations at US\$10252 (US\$30 550) (figure 2B online supplementary table 4). Pharmacy services were US $\$ 5484$ (US\$10 446) for all patients. Similar trends were observed in each severity group, with outpatient visits and hospitalisations remaining the leading cost drivers (figure 2B, online supplementary table 4).

\section{DISCUSSION}

This study characterised a cohort of US patients with newly diagnosed SLE across the spectrum of disease severity, describing patient demographics and clinical characteristics, medication use and the economic burden of SLE. Our findings show that healthcare costs increase in the year before SLE diagnosis and are associated with SLE severity. A similar trend was apparent in the year after diagnosis, when HCRU and costs were shown to increase with increasing disease severity. To our knowledge, previous studies have not evaluated costs in adult US patients during 1-year periods both before and after diagnosis and analysed costs by disease severity.

SLE diagnosis may require an extended period between first symptom onset and official diagnosis, estimated across two studies as a mean of 21.8 months or median of 26.4 months. ${ }^{23}{ }^{29}$ Multiple physician and specialist visits may be involved, ${ }^{30}$ which may be associated with high healthcare costs. Our present findings demonstrate that significant costs are incurred during the year preceding SLE diagnosis, with higher costs among patients who were subsequently diagnosed with more severe disease. Our results follow a similar trend to that reported in a Canadian study that found direct healthcare costs per patient with SLE increased by $97 \%$ in the year preceding diagnosis, after rising by $35 \%$ annually in the 5 years before diagnosis. ${ }^{25}$ The results in McCormick et al ${ }^{25}$ were not stratified by disease severity; therefore, we do not know whether these costs were driven by patients subsequently diagnosed with severe disease, as was the case in our study, or whether patients with mild disease take longer to be diagnosed and therefore incur the largest costs more than 1 year before diagnosis.

In these analyses, we classified SLE disease severity using a claims-based algorithm, ${ }^{9}$ categorising 26.3\% of patients as having mild, $51.0 \%$ moderate and $22.7 \%$ severe SLE over the year after their initial diagnosis. This distribution of SLE severity is consistent with a previous study that developed this algorithm using a different commercial claims dataset ${ }^{9}$ and similar to observations in clinical practice. ${ }^{1722}$ Other studies have used a different algorithm or different time period. For example, Clarke $e t$ al classified SLE severity during the 6-month period after index in a commercially and Medicaid-insured cohort using claims-based data and identified a similar proportion of patients with moderate/severe SLE (commercial: $67.4 \%$; Medicaid: $74.8 \%$ ) or mild SLE (commercial: 
Table 3 Factors associated with total all-cause healthcare costs during the 1-year post-diagnosis (follow-up) period for patients with newly diagnosed SLE: multivariable regression model analysis

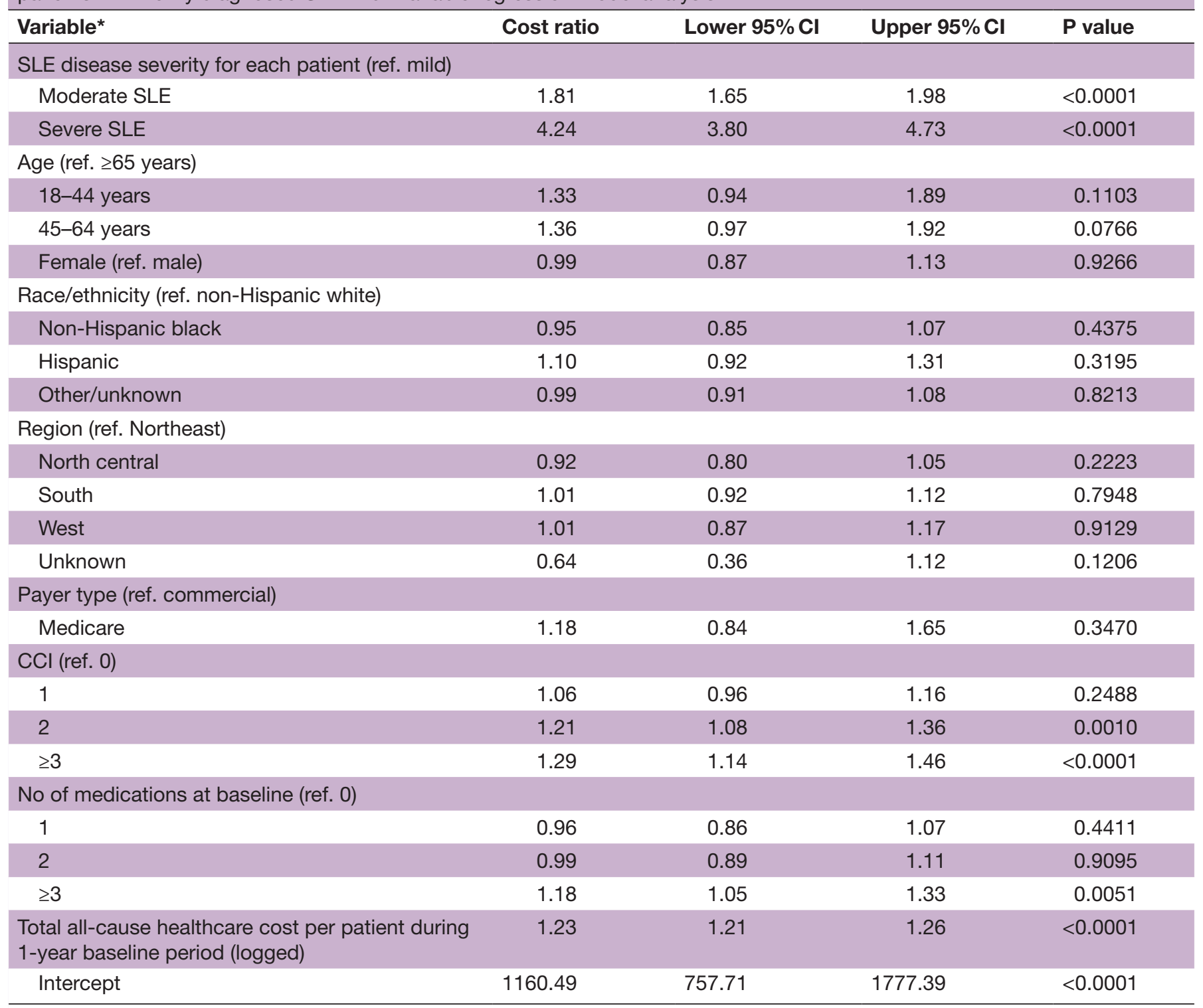

*Generalised linear models with gamma distribution and log transformation.

$\mathrm{CCl}$, Charlson Comorbidity Index; ref., reference.

32.6\%; Medicaid: $25.2 \%$ ) to our study. ${ }^{13}$ The consistency of our findings with other independent cohorts and with results seen in clinical practice provide further support for the use of claims-based algorithms in assessing disease severity by proxy in SLE observational studies where clinical measures of disease severity are not available.

Unadjusted all-cause healthcare costs during the year after diagnosis were 2.2-fold higher for patients with severe SLE and 5.1-fold higher for patients with moderate SLE than for mild SLE. After adjusting for baseline demographics and clinical characteristics, CCI and costs during the baseline period, healthcare costs during the first year post-diagnosis were $81 \%$ higher for moderate SLE and $324 \%$ higher for severe SLE compared with mild SLE. Although there is an increasing body of evidence that severe SLE is associated with higher costs up to 3 years post-diagnosis compared with milder disease, ${ }^{391113-15}$ the present analysis showed that this association is evident as early as the first year after diagnosis. ${ }^{25}$

The largest cost drivers for all patients were outpatient visits and inpatient hospitalisations, consistent with previous studies. ${ }^{9} 11$ 13-15 2531 These cost drivers were the top 2 HCRU categories across all disease severity groups; however, their contribution was greatest for patients with severe SLE. In our study, outpatient visits included injections of SLE-related medications and dialysis, which are costly and may be more frequently associated with severe SLE. Combined outpatient visits and inpatient hospitalisations made up $77 \%$ of the total average costs for patients with severe SLE, compared with $65 \%$ and $61 \%$ for those with moderate and mild SLE, respectively. This result is consistent with the overall study findings and shows that 
while the largest cost drivers were observed across disease severity categories, the contribution of the various cost drivers increased with increasing SLE severity in the year after diagnosis.

The present study identified multiple factors, including the presence of $\geq 2 \mathrm{CCI}$ comorbidities at baseline, the use of $\geq 3$ medications at baseline and higher healthcare costs during the baseline period, that are associated with increased healthcare costs during the year after diagnosis. Previous findings identified association of several of these factors with organ damage progression in patients with SLE. ${ }^{32-34}$ CCI comorbidities and hypertension (a nonCCI comorbidity) are associated with increased organ damage risk. ${ }^{32}{ }^{33}$ Long-term and high-dose corticosteroid use is also a risk factor for organ damage ${ }^{32-34}$ Organ damage may increase healthcare costs and, perhaps most importantly, mortality. ${ }^{32}{ }^{35-37}$ When taken together, these factors, which are associated with both SLE cost and organ damage, may serve as proxies for long-term outcomes and mortality.

Strengths of this study include that it was conducted within the IBM MarketScan commercial claims database, a large and comprehensive data source providing a complete and long-term view of the patient journey in real-world settings that was linked to EMR data. This enabled us to explore additional measures, such as race/ethnicity, for a more comprehensive picture of the patient population. Previous studies were limited in this regard by only having access to a single data source. ${ }^{9} 1016$ The present study also analysed healthcare costs in the year before and after diagnosis, which was previously only reported in one Canadian cohort study. Another study strength is the adjusted costs analysis during the year after diagnosis, which accounts for variables in the year before diagnosis, including healthcare costs and comorbidities. This approach allowed us to adequately assess the drivers of SLE healthcare costs.

A limitation is that our study population was largely commercially insured $(87.7 \%)$. Patients with Medicare supplemental insurance were only $12.3 \%$ of the population, and no Medicaid patients were included. However, linking claims and EMR data ensured that we comprehensively captured SLE-related HCRU and costs and that our study cohort was similar to studies that used commercially and Medicare insured study populations. ${ }^{13} 15$ Another limitation involves potential misclassification using a claims-based algorithm, both in identifying newly diagnosed patients with SLE and classifying them into appropriate disease severity groups, because HCRU was used to classify SLE severity and to calculate costs. However, the distribution of severity was similar to that observed in clinical practice and we supplemented the claims-based algorithm with EMR data to further reduce any potential misclassification or bias. Finally, indirect costs such as diminished work and non-work productivity, and caregiver burden are not captured in the linked database. Indirect costs may be substantial for patients with SLE. Studies estimate that indirect costs exceed direct costs by up to 2- to 4 -fold. ${ }^{38}$ Thus, the full economic burden of SLE is likely to be much higher than the direct costs reported in our study.

In conclusion, this retrospective real-world study of US patients with newly diagnosed SLE demonstrates that moderate and severe SLE was associated with higher HCRU and all-cause healthcare costs in the 1-year period after diagnosis compared with mild SLE. Baseline comorbidities and all-cause healthcare costs were also higher among patients with moderate and severe SLE during the year before diagnosis. These findings highlight that early diagnosis, and treatments to achieve disease control, may improve health outcomes and reduce the economic burden of SLE.

Acknowledgements Writing and editing assistance was provided by Zachary Harrelson, PhD, and Sarah Thornburg, of JK Associates Inc., part of Fishawack Health. This support was funded by AstraZeneca.

Contributors ERH, BD, XW and MJ conceived and designed the study. AMN acquired the data and designed the study. All authors analysed and interpreted the data. All authors were involved in development, review and final approval of the manuscript.

Funding Analysis supported by AstraZeneca.

Competing interests MJ, BD, XW and ERH are employees of AstraZeneca. AMN is an employee of IQVIA and was paid by AstraZeneca to conduct this research study. Patient consent for publication Not required.

Provenance and peer review Not commissioned; externally peer reviewed. Data availability statement Data are available upon reasonable request. Data underlying the findings described in this manuscript may be obtained in accordance with AstraZeneca's data sharing policy described at https://astrazenecagrouptrials. pharmacm.com/ST/Submission/Disclosure. This study is based on de-identified data obtained and analysed by IQVIA under license from IBM MarketScan and GE Healthcare, and so are not publicly available. The interpretation and conclusions contained in this study are those of the authors alone. The authors do not own these data and hence are not permitted to share the data in the original form.

Supplemental material This content has been supplied by the author(s). It has not been vetted by BMJ Publishing Group Limited (BMJ) and may not have been peer-reviewed. Any opinions or recommendations discussed are solely those of the author(s) and are not endorsed by BMJ. BMJ disclaims all liability and responsibility arising from any reliance placed on the content. Where the content includes any translated material, BMJ does not warrant the accuracy and reliability of the translations (including but not limited to local regulations, clinical guidelines, terminology, drug names and drug dosages), and is not responsible for any error and/or omissions arising from translation and adaptation or otherwise.

Open access This is an open access article distributed in accordance with the Creative Commons Attribution Non Commercial (CC BY-NC 4.0) license, which permits others to distribute, remix, adapt, build upon this work non-commercially, and license their derivative works on different terms, provided the original work is properly cited, appropriate credit is given, any changes made indicated, and the use is non-commercial. See: http://creativecommons.org/licenses/by-nc/4.0/.

\section{REFERENCES}

1 Mok CC, Lau CS. Pathogenesis of systemic lupus erythematosus. $J$ Clin Pathol 2003;56:481-90.

2 Kalunian K, Merrill JT. New directions in the treatment of systemic lupus erythematosus. Curr Med Res Opin 2009;25:1501-14.

3 Hammond E, Murimi I, Lin D. SAT0227 health care utilization and costs of systemic lupus erythematosus (SLE) in the United States: systematic review. Ann Rheum Dis 2017;76:859-60.

4 Panopalis P, Yazdany J, Gillis JZ, et al. Health care costs and costs associated with changes in work productivity among persons with systemic lupus erythematosus. Arthritis Rheum 2008;59:1788-95.

5 Silverman S, Dukes EM, Johnston SS, et al. The economic burden of fibromyalgia: comparative analysis with rheumatoid arthritis. Curr Med Res Opin 2009;25:829-40. 
6 Petri M, Singh S, Tesfasyone $\mathrm{H}$, et al. Prevalence of flare and influence of demographic and serologic factors on flare risk in systemic lupus erythematosus: a prospective study. $J$ Rheumatol 2009;36:2476-80.

7 Mirzayan MJ, Schmidt RE, Witte T. Prognostic parameters for flare in systemic lupus erythematosus. Rheumatology 2000;39:1316-9.

8 Narayanan S, Wilson K, Ogelsby A, et al. Economic burden of systemic lupus erythematosus flares and comorbidities in a commercially insured population in the United States. J Occup Environ Med 2013;55:1262-70.

9 Garris C, Jhingran P, Bass D, et al. Healthcare utilization and cost of systemic lupus erythematosus in a US managed care health plan. $J$ Med Econ 2013;16:667-77.

10 Kan HJ, Song X, Johnson BH, et al. Healthcare utilization and costs of systemic lupus erythematosus in Medicaid. Biomed Res Int 2013;2013:1-8.

11 Tanaka Y, Mizukami A, Kobayashi A, et al. Disease severity and economic burden in Japanese patients with systemic lupus erythematosus: a retrospective, observational study. Int $J$ Rheum Dis 2018;21:1609-18.

12 Mosca M, Tani C, Carli L, et al. Glucocorticoids in systemic lupus erythematosus. Clin Exp Rheumatol 2011;29:S126-9.

13 Clarke AE, Yazdany J, Kabadi SM, et al. The economic burden of systemic lupus erythematosus in commercially- and medicaidinsured populations in the United States. Semin Arthritis Rheum 2020;50:759-68.

14 Miyazaki C, Sruamsiri R, Mahlich J, et al. Treatment patterns and medical cost of systemic lupus erythematosus patients in Japan: a retrospective claims database study. J Med Econ 2020;23:786-99.

15 Murimi-Worstell IB, Lin DH, Kan H, et al. Healthcare utilization and costs of systemic lupus erythematosus by disease severity in the United States. J Rheumatol 2021;48:385-93.

16 Shah M, Chaudhari S, McLaughlin TP, et al. Cumulative burden of oral corticosteroid adverse effects and the economic implications of corticosteroid use in patients with systemic lupus erythematosus. Clin Ther 2013;35:486-97.

17 Doria A, Amoura Z, Cervera R, et al. Annual direct medical cost of active systemic lupus erythematosus in five European countries. Ann Rheum Dis 2014;73:154-60.

$18 \mathrm{Kan} \mathrm{H}$, Nagar S, Patel J, et al. Longitudinal treatment patterns and associated outcomes in patients with newly diagnosed systemic lupus erythematosus. Clin Ther 2016;38:610-24.

19 Conti F, Ceccarelli F, Perricone C, et al. The chronic damage in systemic lupus erythematosus is driven by flares, glucocorticoids and antiphospholipid antibodies: results from a monocentric cohort. Lupus 2016;25:719-26.

20 Zonana-Nacach A, Barr SG, Magder LS, et al. Damage in systemic lupus erythematosus and its association with corticosteroids. Arthritis Rheum 2000;43:1801-8.

21 Chen S-Y, Choi C-B, Li Q, et al. Glucocorticoid use in patients with systemic lupus erythematosus: association between dose and health care utilization and costs. Arthritis Care Res 2015;67:1086-94.
22 Clarke AE, Urowitz MB, Monga N, et al. Costs associated with severe and nonsevere systemic lupus erythematosus in Canada. Arthritis Care Res 2015;67:431-6.

23 Ozbek S, Sert M, Paydas S, et al. Delay in the diagnosis of SLE: the importance of arthritis/arthralgia as the initial symptom. Acta Med Okayama 2003;57:187-90.

24 Oglesby A, Korves C, Laliberté F, et al. Impact of early versus late systemic lupus erythematosus diagnosis on clinical and economic outcomes. Appl Health Econ Health Policy 2014;12:179-90.

25 McCormick N, Marra CA, Sadatsafavi M, et al. Incremental direct medical costs of systemic lupus erythematosus patients in the years preceding diagnosis: a general population-based study. Lupus 2018;27:1247-58.

26 Zubeldia K, Romney GW. Inventors, Romney GW, assignee. Anonymously linking a plurality of data records. US patent 6397224 2002.

27 Ober NS, Grubmuller J, Farrell M. System and method for generating de-identified health care data. US patent 67321132004

28 Ober NS, Grubmuller J, Farrell M. System and method for generating de-identified health care data. US patent 73766772008

29 Nightingale AL, Davidson JE, Molta CT, et al. Presentation of SLE in UK primary care using the Clinical Practice Research Datalink. Lupus Sci Med 2017:4:e000172.

30 Kuhn A, Bonsmann G, Anders H-J, et al. The diagnosis and treatment of systemic lupus erythematosus. Dtsch Arztebl Int 2015;112:423-32.

31 Kariburyo F, Xie L, Sah J, et al. Real-world medication use and economic outcomes in incident systemic lupus erythematosus patients in the United States. J Med Econ 2020;23:1-9.

32 Bruce IN, O'Keeffe AG, Farewell V, et al. Factors associated with damage accrual in patients with systemic lupus erythematosus: results from the Systemic Lupus International Collaborating Clinics (SLICC) inception cohort. Ann Rheum Dis 2015;74:1706-13.

33 Kim S-K, Choe J-Y, Lee S-S. Charlson comorbidity index is related to organ damage in systemic lupus erythematosus: data from KORean lupus Network (KORNET) registry. J Rheumatol 2017;44:452-8.

34 Tarr T, Papp G, Nagy N, et al. Chronic high-dose glucocorticoid therapy triggers the development of chronic organ damage and worsens disease outcome in systemic lupus erythematosus. Clin Rheumatol 2017;36:327-33.

35 Furst DE, Clarke A, Fernandes AW, et al. Resource utilization and direct medical costs in adult systemic lupus erythematosus patients from a commercially insured population. Lupus 2013;22:268-78.

36 Jönsen A, Bengtsson AA, Hjalte F, et al. Total cost and cost predictors in systemic lupus erythematosus - 8-years follow-up of a Swedish inception cohort. Lupus 2015;24:1248-56.

37 Jönsen A, Hjalte F, Willim M, et al. Direct and indirect costs for systemic lupus erythematosus in Sweden. A nationwide health economic study based on five defined cohorts. Semin Arthritis Rheum 2016;45:684-90.

38 Carter EE, Barr SG, Clarke AE. The global burden of SLE: prevalence, health disparities and socioeconomic impact. Nat Rev Rheumatol 2016;12:605-20. 